\title{
Special issue "Science of solar system materials examined from Hayabusa and future missions (II)"
}

\author{
Tatsuaki Okada ${ }^{*}$, Richard P. Binzel ${ }^{2}$, Harold C. Connolly Jr. ${ }^{3}$, Toru Yada ${ }^{1}$ and Keiji Ohtsuki ${ }^{4}$
}

Six years have passed since the first asteroid sample was returned from the S-type near-Earth asteroid 25143 Itokawa by the JAXA's Hayabusa mission in 2010 (Yada et al. 2014). Considerable progress has been made in the study of surface regolith materials and the understanding of planetary surface processes such as space weathering (Noguchi et al. 2011), the chronology of Itokawa and its dynamic evolution processes (Nagao et al. 2011; Park et al. 2015), and the thermal alteration undergone in parent bodies (Nakamura $T$ et al. 2011). Discussions of new findings from the Hayabusa-returned samples and from a large collection of meteorites, micrometeorites, and interplanetary dust particles have continued, especially at the annual international Hayabusa symposia of solar system materials (Okada et al. 2015). Progress in sample return science has driven the next stage of exploration. Now, two new sample return missions to primitive, volatile-rich asteroids, JAXA's Hayabusa2 (Tsuda et al. 2013) and NASA's OSIRIS-REx (Lauretta et al. 2012), are en route to their target bodies, C-type 162173 Ryugu and B-type 101955 Bennu, respectively.

It is our great pleasure to present our second special issue of the journal Earth, Planets and Space, "Science of solar system materials examined from Hayabusa and future missions (II)." This special issue is based on discussions during the Hayabusa 2014 symposium, which featured new results from Hayabusa-returned samples and related studies, but was also open to any scientific results regarding primitive bodies and the early solar system, the results of laboratory experiments and ground-based

\footnotetext{
*Correspondence: okada@planeta.sci.isas.jaxa.jp

${ }^{1}$ Institute of Space and Astronautical Science, Japan Aerospace

Exploration Agency, Sagamihara 252-5210, Japan

Full list of author information is available at the end of the article
}

observations, and reports of new instruments and methods. We will begin with a brief introduction to the missions of the Hayabusa and its successor Hayabusa2. In addition, all six manuscripts published in this special issue are reviewed below.

Hayabusa (formerly MUSES-C) was an engineering mission organized by the Japan Aerospace Exploration Agency (JAXA) to demonstrate the technology to return samples from a near-Earth asteroid (Kawaguchi et al. 2003). It was launched in 2003, explored the S-type asteroid 25143 Itokawa in 2005, and returned surface samples from the asteroid to Earth in 2010. The four main technologies demonstrated in this mission during its roundtrip to the asteroid were the ion engine used as the main thrusters, the optical navigation for approaching and landing on the asteroid, the sample collection on the asteroid under microgravity conditions, and the hypervelocity reentry of the sample capsule into the terrestrial atmosphere. These technical challenges were all successfully performed, and the asteroid samples were returned to Earth. This mission also has scientific significance for elucidating the origin and evolution of the early solar system based on exploration of a primitive solar system body. We found that the small S-type asteroid 25143 Itokawa was chondritic in composition and a low-density rubble pile in structure (e.g., Fujiwara et al. 2006). Most of its surface was rough and covered with boulders, but a small portion of its surface, where the samples were collected, was smooth and covered with pebbles (e.g., Yano et al. 2006). The returned samples have been curated in JAXA's Extraterrestrial Sample Curation Center, organized by the Astromaterials Science Research Group, Institute of Space and Astronautical Science, JAXA (Yada et al. 2014). About 700 grains have been cataloged thus 
far, with information from optical microscopy, scanning electron microscopy (SEM), and energy-dispersive X-ray spectroscopy (EDS). Parts of the catalogued samples are available to researchers if their research proposals are accepted through a peer-review process. The results of primitive examinations of the Itokawa samples just after they were returned, as well as data from continued studies of these samples, have been reported in past special issues (e.g., Nakamura T et al. 2011; Nakamura E et al. 2012). A portion of these detailed studies was introduced in the previous EPS special issue "Science of solar system materials examined by Hayabusa and future missions."

Hayabusa2, the second asteroid sample return mission by JAXA, was launched in 2014 and is now on its way to the asteroid 162173 Ryugu. The target asteroid is classified as C-type, a volatile-rich carbonaceous type. The technology of Hayabusa2 is based on that of the first Hayabusa mission, but its science experiments are more specified to C-type asteroid and reinforced with the instruments (Tachibana et al. 2014). The remote sensing instruments are designed to focus on investigating the degree of hydration of minerals by measuring $3-\mu \mathrm{m}$ band absorption features and on physical state of the surface, such as the thermo-physical properties of the asteroid based on thermal infrared imaging (Okada and Iwata 2014). The spacecraft has a scientific lander called MASCOT (Micro-Asteroid Surface Scout) to conduct observations in situ on the surface of the asteroid (Ho et al. 2016). In the Hayabusa 2 mission, scientific measurements will be conducted at a global scale with remote sensing, a local scale with the surface lander, and a microscale through analysis of returned samples. In addition, an impact experiment will be conducted using a small carry-on impactor; the excavated fresh, unweathered materials will be observed via remote sensing, and those materials will be returned to Earth.

A great deal of new results from the Hayabusareturned samples has been presented at the annual Hayabusa symposia. In this special issue, another new finding from microscopic analysis has been provided by Dobrică and Ogliore (2016). This highlighted paper reports new microstructures on micron-scale particles. These researchers investigated the surface textures and chemical compositions of two small particles (RB-DQ040062 and RB-DQ04-0091) returned from the surface of the asteroid Itokawa by Hayabusa. Using high-resolution images from scanning electron, helium ion, and scanning transmission electron microscopy, they identified splash melts, surface blistering, and numerous small adhering particles on the Itokawa samples. They extracted seven focused ion beam sections from both of these Itokawa particles, targeting one splash melt and ten adhering particles, to investigate their composition and provenance and the role of micrometeoroid impacts on Itokawa's surface. Based on the structure, mineralogy, and interface between the adhering particles and host grains, lithic fragments and particles deposited by impact were identified. These particles have morphologies and compositions consistent with impact-generated deposits, such as impact-generated silica glass, and one was a Ni-free, metallic Fe-rich, and S-rich assemblage that was likely generated by vapor recondensation during a micrometeoroid impact. This study shows that even though the regolith of the asteroid Itokawa is young (Nagao et al. 2011; Noguchi et al. 2011), it has been altered by micrometeoroid impacts.

Volatile-rich carbonaceous meteorites are increasingly attracting the interest of researchers to determine the implications of the properties of these meteorites to future missions such as Hayabusa2 and OSIRIS-REx. King et al. (2015) performed laboratory experiments to constrain the thermal alteration processes of volatilerich asteroids. CI and CI-like carbonaceous chondrites provide a record of aqueous alteration in the early solar system, but those of the latter type seem to have experienced a late-stage period of thermal metamorphism. To constrain the nature and extent of the aqueous and thermal alteration, the bulk mineralogy and abundance of $\mathrm{H}_{2} \mathrm{O}$ in CI chondrites (Ivuna and Orgueil) and CI-like chondrites (Y-82162 and Y-980115) were investigated using thermo-gravimetric analysis as well as near- and mid-infrared spectroscopy. Both CI chondrites analyzed showed significant loss of mass $(\sim 30 \mathrm{wt} \%)$ and similar patterns of mass loss upon heating to $1000{ }^{\circ} \mathrm{C}$ due to dehydration and dehydroxylation of abundant phyllosilicates and Fe-(oxy)hydroxides, as well as the decomposition of Fe sulfides, carbonates, and organics. Infrared spectra of both CI chondrites have prominent 3- $\mu \mathrm{m}$ features because of bound $\mathrm{OH}^{-} / \mathrm{H}_{2} \mathrm{O}$ in phyllosilicates and $\mathrm{Fe}$-(oxy)hydroxides and only minor $11-\mu \mathrm{m}$ features from anhydrous silicates. These results indicate complete aqueous alteration and nearly the same degree of hydration experienced in both $\mathrm{CI}$ meteorites. In contrast, both of the CI-like chondrites show lower mass loss and contain less than a half the $\mathrm{H}_{2} \mathrm{O}$ of the $\mathrm{CI}$ chondrites, and the $3-\mu \mathrm{m}$ feature is nearly absent from spectra, whereas the $11-\mu \mathrm{m}$ feature is intense. These CI-like chondrites experienced thermal metamorphism at temperatures $>500^{\circ} \mathrm{C}$, which initially caused dehydration and dehydroxylation of phyllosilicates. Concerning future sample return missions, King et al. (2015) also note that the surfaces of many $\mathrm{C}$-type asteroids have likely been heated through impact metamorphism and/or solar radiation and that thermally altered carbonaceous chondrites may therefore be good analogues for the samples that will be returned by the Hayabusa2 and OSIRIS-REx missions. 
Chan et al. (2016) developed a novel method using gas chromatography, combustion, and isotope ratio mass spectrometry to determine compound-specific nitrogen isotope compositions with an achiral column, which was previously shown to offer high precision for nitrogen isotopic analysis. They applied this method to determine the amino acid contents and stable nitrogen isotopic compositions of individual amino acids from thermally metamorphosed (above $500{ }^{\circ} \mathrm{C}$ ) Antarctic carbonaceous chondrites, specifically the Ivuna-like (CI-like) meteorite Y-980115 and the Ornans-like (CO3.5) meteorite ALHA77003. In ALHA77003, almost no amino acids were detected because of its extensive history of thermal alteration. In Y-980115, amino acids were identified, and the $\delta^{15} \mathrm{~N}$ values of selected amino acids are clearly extraterrestrial. Y-980115 has experienced an extended period of aqueous alteration, as indicated by the presence of hydrous mineral phases. This meteorite has also been exposed to at least one short-lived period of thermal metamorphism post-hydration. Chan et al. (2016) also suggest that these carbonaceous chondrite samples are good analogues for the target asteroid of the Hayabusa2 mission, Ryugu, which is a C-type asteroid likely composed of heterogeneous materials including both hydrated and dehydrated minerals.

In Hayabusa, Hayabusa2, OSIRIS-REx, and other missions, infrared spectroscopy helps us investigate surface mineralogy, the degree of hydration of silicates, and the existence of organics. Many databases have been constructed based on laboratory experiments to calibrate and interpret the observational results. However, in many cases, these experiments have been conducted under conditions too simplified to apply findings to real observations. Maturilli et al. (2016a) have developed and organized the Planetary Emissivity Laboratory (PEL) of the German Aerospace Center (DLR) and have demonstrated in laboratory experiments the dependence of laboratory-measured emissivity spectra on the emergence angle. Most of the previous work has been based on reflectance measurements in the visible to near-infrared spectral region, on emissivity measurements of flat, solid surfaces, and on measurements from the nadir direction, which are not directly applicable to the analysis of remote sensing data. Small bodies such as the asteroid Itokawa (the target of the Hayabusa mission) have very irregular surfaces, which is likely also the case for the asteroids Ryugu and Bennu, the targets of the Hayabusa2 and the OSIRIS-REx missions, respectively. The spectra from such rough surfaces are difficult to compare with previously reported laboratory spectra. Maturilli et al. (2016a) set up a series of spectral measurements to investigate this problem in the 1- to $16-\mu \mathrm{m}$ spectral range and measured the emissivity of two asteroid analogue materials (meteorite Millbillillie and a synthetic enstatite) and slabs of serpentinite and basalt in a vacuum and under purged air, at a surface temperature of $100{ }^{\circ} \mathrm{C}$, for emergence angles from $0^{\circ}$ to $60^{\circ}$. Complementary reflectance measurements of the four samples were collected. For all samples measured, they found that emissivity and reflectance both show the same trend: Significant variations from values obtained at the nadir $\left(0^{\circ}\right.$ emergence angle) appear only for emergence angles $\geq 40^{\circ}$.

Maturilli et al. (2016b) also constructed a new big database of emissivity and reflectance spectra that characterize asteroid analogues measured under vacuum conditions. A number of missions, such as NEAR-Shoemaker, Hayabsua, Rosetta, Hayabusa2, and OSIRIS-Rex, have targeted small bodies in the solar system. Despite the rich collection of reflectance and emissivity spectral libraries available for asteroid analogues, these data are mainly based on measurements in air for a spectral range covering the visible to the mid-infrared spectrum from 0.4 to $25 \mu \mathrm{m}$. To characterize minerals, rocks and meteorites suitable to serve as surface analogues for asteroids and cometary nuclei in general, spectroscopic measurements are needed for a wider spectral range and under vacuum conditions. To fill this gap, these authors acquired spectral measurements over a large spectral range $(1-100 \mu \mathrm{m})$ for several meteorites and other analogues at the PEL of DLR in Berlin. These data provide more direct and suitable analogues for emissivity and reflectance measurements of asteroid and cometary surfaces.

At the annual Hayabusa symposia, instrumentation and methodology are essential topics for answering unresolved questions. Remote X-ray spectroscopy is one of the main instrumental techniques used in missions to terrestrial bodies. Hong and Romaine (2016) have developed sophisticated new X-ray optics that will be applied in remote planetary $\mathrm{X}$-ray imaging spectrometry. The compositions of diverse planetary bodies are of fundamental interest to planetary science; they provide clues about the formation and evolutionary history of target bodies and the solar system. The masses and volumes of instruments used in focusing X-ray optics have been too large for resource-limited in situ missions; therefore, near-target X-ray observations of planetary bodies have been limited to those of simple collimator-type X-ray instruments. However, Hong and Romaine (2016) have now introduced the new Miniature lightweight WolterI focusing X-ray Optics (MiXO), which uses metalceramic hybrid X-ray mirrors based on electroformed nickel replication and plasma thermal spray processes. MiXO enables compact, powerful imaging by X-ray telescopes suitable for future planetary missions, especially for observing relatively small planetary bodies such as 
asteroids and comet nuclei. These researchers have also presented several example configurations of MiXO telescopes and have demonstrated their performance relative to an alternative approach, micropore optics, which is being employed for the first planetary focusing X-ray telescope, the Mercury Imaging X-ray Spectrometer-T onboard BepiColombo. X-ray imaging spectroscopy using MiXO will open a wide area for new discoveries in planetary science and will greatly enhance our understanding of the natures and origins of diverse planetary bodies.

All manuscripts in this special issue are fruitful and constructive for the ongoing discussion of the origin and evolution of the early solar system, and for the prospects of future volatile-rich carbonaceous-type asteroid missions such as Hayabusa2 and OSIRIS-REx, which will soon reach their destinations and commence observations, and then return abundant samples to Earth. Furthermore, these activities provide motivation for new missions to yet unvisited types of primitive bodies, which has led to NASA's selection of the Psyche mission for rendezvous with a metallic core (Elkins-Tanton et al. 2016) and the Lucy mission for multiple flybys of Trojan asteroids (Levison et al. 2016), and will hopefully lead to landing on a Trojan asteroid in a future mission (Okada et al. 2017).

\section{Authors' contributions}

TO is the lead guest editor of this special issue. RPB, HCC, TY, KO serve as the guest editors of this special issue. All authors read and approved the final manuscript.

\section{Author details \\ ${ }^{1}$ Institute of Space and Astronautical Science, Japan Aerospace Exploration Agency, Sagamihara 252-5210, Japan. ${ }^{2}$ Earth, Atmospheric and Planetary Sciences, Massachusetts Institute of Technology, Cambridge, MA 02139-4307, USA. ${ }^{3}$ Department of Earth and Environmental Sciences, Kingsborough Com- munity College of the City University of New York, Brooklyn, NY 11235-2398, USA. ${ }^{4}$ Department of Earth and Planetary Sciences, Kobe University, Kobe 657-8501, Japan.}

Received: 5 February 2017 Accepted: 5 February 2017

Published online: 13 February 2017

\section{References}

Chan QHS, Chikaraishi Y, Takano Y, Ogawa NO, Ohkouchi N (2016) Amino acid compositions in heated carbonaceous chondrites and their compoundspecific nitrogen isotopic ratios. Earth Planets Space 68:7. doi:10.1186/ s40623-016-0382-8

Dobrică E, Ogliore RC (2016) Adhering grains and surface features on two Itokawa particles. Earth Planets Space 68:21. doi:10.1186/ s40623-016-0391-7

Elkins-Tanton LT, Asphaug E, Bell J, Bercovici D, Billis BG, Binzel RP, Bottke WF, Goldsten J, Jaumann R, Jun I, Lawrence DJ, Marchi S, Oh D, Park R, Peplowski PN, Polanskey CA, Prettyman TH, Raymond CA, Russel CT, Weiss BP, Wenkert DD, Wieczorek M, Zuber MT (2016) Asteroid (16) psyche: the science of visiting a metal world. Lunar Planet Sci Conf 47:\#1631

Fujiwara A, Kawaguchi J, Yeomans DK, Abe M, Mukai T, Okada T, Saito J, Yano H, Yoshikawa M, Scheeres DJ, Barnouin-Jha O, Cheng AF, Demura H, Gaskell RW, Hirata N, Ikeda H, Kominato T, Miyamoto H, Nakamura AM, Nakamura
R, Sasaki S, Uesugi K (2006) The rubble-pile asteroid Itokawa as observed by Hayabusa. Science 312:1330-1334

Ho TM, Baturkin V, Grimm C, Grundmann JT, Hobbie C, Ksenik E, Lange C, Sasaki K, Schlotterer M, Talapina M, Termtanasombat N, Wejmo E, Witte L, Wrasmann M, Wuebbels G, Roessler J, Ziach C, Findlay R, Biele J, Krause C, Ulamec S, Lange M, Mierheim O, Lichtenheldt R, Maier M, Reill J, Sedlmayr $\mathrm{HJ}$, Bousquet P, Bellion A, Bompis O, Cenac-Morthe C, Deleuze M, Fredon S, Jurado E, Canalias E, Jaumann R, Bibring JP, Glassmeier KH, Hercik D, Grott M, Celotti L, Cordero F, Hendrikse J, Okada T (2016) MASCOT: the mobile asteroid surface scout onboard the Hayabusa2 mission. Space Sci Rev. doi:10.1007/s11214-016-0251-6

Hong J, Romaine S (2016) Miniature lightweight X-ray optics (MiXO) for surface elemental composition mapping of asteroids and comets. Earth Planets Space 68:35. doi:10.1186/s40623-016-0409-1

Kawaguchi J, Uesugi K, Fujiwara A (2003) The MUSES-C mission for the sample and return -its technology development status and readiness. Acta Astronaut 52:117-123

King AJ, Solomon JR, Schofield PF, Russell SS (2015) Characterising the Cl and $\mathrm{Cl}$-like carbonaceous chondrites using thermogravimetric analysis and infrared spectroscopy. Earth Planets Space 67:198. doi:10.1186/ s40623-015-0370-4

Lauretta DS, The OSIRIS-REx Team (2012) An overview of the OSIRIS-REx asteroid sample return mission. Lunar Planet Sci Conf 43:\#2491

Levison HF, Team Lucy Science (2016) Lucy: surveying the diversity of the Trojan asteroids, the fossils of planet formation. Lunar Planet Sci Conf 47:2061

Maturilli A, Helbert J, Ferrari S, D'Amore M (2016a) On the effect of emergence angle on emissivity spectra: application to small bodies. Earth Planets Space 68:84. doi:10.1186/s40623-016-0464-7

Maturilli A, Helbert J, Ferrari S, Davidsson B, D'Amore M (2016b) Characterization of asteroid analogues by means of emission and reflectance spectroscopy in the 1- to 100- $\mu \mathrm{m}$ spectral range. Earth Planets Space 68:113. doi:10.1186/s40623-016-0489-y

Nagao K, Okazaki R, Nakamura T, Miura YN, Osawa T, Bajo K, Matsuda S, Ebihara M, Ireland TR, Kitajima F, Naraoka H, Noguchi T, Tsuchiyama A, Yurimoto H, Zolensky ME, Uesugi M, Shirai K, Abe M, Yada T, Ishibashi Y, Fujimura A, Mukai T, Ueno M, Okada T, Yoshikawa M, Kawaguchi J (2011) Irradiation history of Itokawa regolith material deduced from noble gases in the Hayabusa samples. Science 333:1128-1131

Nakamura T, Noguchi T, Tanaka M, Zolensky ME, Kimura M, Tsuchiyama A, Nakato A, Ogami T, Ishida H, Uesugi M, Yada T, Shirai S, Fujimura A, Okazaki R, Sandford SA, Ishibashi Y, Abe M, Okada T, Ueno M, Mukai T, Yoshikawa M, Kawaguchi J (2011) Itokawa dust particles: a direct link between S-type asteroids and ordinary chondrites. Science 333:1113-1116

Nakamura E, Makishima A, Moriguti T, Kobayashi K, Tanaka R, Kunihiro T, Tujimori T, Sakaguchi C, Kitagawa H, Ota T, Yachi Y, Yada T, Abe M, Fujimura A, Ueno M, Mukai T, Yoshikawa M, Kawaguchi J (2012) Space environment of and asteroid preserved on micrograins returned by the Hayabusa spacecraft. Proc Natl Acad Sci. doi:10.1073/pnas.1116236109

Noguchi T, Nakamura T, Kimura M, Zolensky ME, Tanaka M, Hashimoto T, Konno M, Nakato A, Ogami T, Fujimura A, Abe M, Yada T, Mukai T, Ueno M, Okada T, Shirai K, Ishibashi Y, Okazaki R (2011) Incipient space weathering observed on the surface of Itokawa dust particles. Science 333:1121-1125

Okada T, Iwata T (2014) Near infrared spectroscopy and thermal infrared imagery on Hayabusa2: hydrated and physical state of C-class asteroid 1999JU3. In: Ip WH (ed) New results in observations and space exploration of asteroids, Proc. Intl. CJMT-1 workshop on asteroidal science, Macao, Oct 16-17, 2012, pp 97-112

Okada T, Zolensky ME, Ireland TR, Yada T (2015) The earth, planets and space special issue: "science of solar system materials examined from Hayabusa and future missions". Earth Planets Space 67:116. doi:10.1186/ s40623-015-0235-x

Okada T, Iwata T, Matsumoto J, Bibring JP, Ulamec S, Jaumann R, Nakamura R, Yano H, Kebukawa Y, Aoki J, Kawai Y, Terada K, Toyoda M, Ito M, Yabuta K, Yurimoto H, Saito Y, Yokota S, Okamoto C, Matsuura S, Tsumura K, Yonetoku D, Mihara T, Matsuoka A, Nomura R, Hirai T, Grand N, Cottin H, Thirkell L, Briois C, Saiki T, Kato H, Mori O, Kawaguchi J (2017) Science and exploration of a Jupiter Trojan asteroid in the Solar Power Sail mission. Lunar Planet Sci Conf 48:\#1828 
Park J, Turrin BD, Herzog GF, Lindsay FN, Delaney JS, Swisher CC, Uesugi M, Karouji Y, Yada T, Abe M, Okada T, Ishibashi Y (2015) ${ }^{40} \mathrm{Ar} /{ }^{39} \mathrm{Ar}$ age of material returned from asteroid 25143 Itokawa. Meteorit Planet Sci. doi:10.1111/maps.12564

Tachibana S, Abe M, Arakawa M, Fujimoto M, lijima Y, Ishiguro M, Kitazato K, Kobayashi N, Namiki N, Okada T, Okazaki R, Sawada H, Sugita S, Takano Y, Tanaka S, Watanabe S (2014) Hayabusa2: scientific importance of samples returned from C-type near-earth asteroid (162173) $1999 \mathrm{JU}_{3}$. Geochem J 48:571-587. doi:10.2343/geochemj.2.0350

Tsuda Y, Yoshikawa M, Abe M, Minamino H, Nakazawa S (2013) System design of the Hayabusa2-asteroid sample return mission to 1999 JU3. Acta Astronaut 91:356-362. doi:10.1016/j.actaastro.2013.06.028
Yada T, Fujimura A, Abe M, Nakamura T, Noguchi T, Okazaki R, Nagao K, Ishibashi Y, Shirai K, Zolensky ME, Sandford S, Okada T, Uesugi M, Karouji Y, Ogawa M, Yakame S, Ueno M, Mukai T, Yoshikawa M, Kawaguchi J (2014) Hayabusa returned sample curation in the planetary material sample curation facility of JAXA. Meteorit Planet Sci 49:135-153

Yano H, Kubota T, Miyamoto H, Okada T, Scheeres D, Takagi Y, Yoshida K, Abe M, Abe S, Barnouin-Jha O, Fujiwara A, Hasegawa S, Hashimoto T, Ishiguro M, Kato M, Kawaguchi J, Mukai T, Saito J, Sasaki S, Yoshikawa M (2006) Touchdown of the Hayabusa spacecraft at the Muses Sea on Itokawa. Science 312:1350-1353

\section{Submit your manuscript to a SpringerOpen ${ }^{\odot}$ journal and benefit from:}

- Convenient online submission

- Rigorous peer review

- Immediate publication on acceptance

- Open access: articles freely available online

- High visibility within the field

- Retaining the copyright to your article 